\title{
A NOTE ON NECESSARY CONDITIONS OF HYPOELLIPTICITY FOR SOME CLASSES OF DIFFERENTIAL OPERATORS WITH DOUBLE CHARACTERISTICS
}

\author{
NGUYEN Minh TRI
}

\begin{abstract}
We construct explicit formulas for fundamental solutions and global non-smooth solutions at degenerate points of some classes of differential operators with double characteristics. A new elementary proof for non-hypoellipticity is given.
\end{abstract}

\section{§1. Introduction}

In this paper we will construct explicit formulas for fundamental solutions and global non-smooth solutions at degenerate points of the following operator

$$
G_{k, c}^{a, b}=X_{2} X_{1}+i c x^{k-1} \frac{\partial}{\partial y}
$$

where $(x, y) \in \boldsymbol{R}^{2} ; a, b, c \in \boldsymbol{C}, \operatorname{Re} a \cdot \operatorname{Re} b \neq 0 ; i=\sqrt{-1} ; k$ is a positive integer, and $X_{1}=(\partial / \partial x)-i b x^{k}(\partial / \partial y), \quad X_{2}=(\partial / \partial x)-i a x^{k}(\partial / \partial y)$. The operator $G_{k, c}^{a, b}$ was studied in [1] when $k$ is odd and in [2] when $k$ is even. For more complete references and generalization we refer to [3], [4], [5], [6], [7] and therein references. We will treat only the case $\operatorname{Re} a<0$. The case $\operatorname{Re} a>0$ can be considered analogously. Recently in [8], [9] we considered a model of the Grushin operator, that is the case when $a=-1, b=1$, and the Kohn-Laplacian on the Heisenberg group. The paper is organized as follows. In $\$ 2$ we give some definitions of notations used in the paper, and establish some auxiliary lemmas. In $\S 3$ we state and prove the main results.

\section{§2. Auxiliary lemmas}

We will use the following notation

$$
(z, m)=z(z+1) \cdots(z+m-1)=\frac{\Gamma(z+m)}{\Gamma(z)} \text { for } z \in C, m \in N .
$$

1991 Mathematics Subject Classification: Primary 35H05, Secondary 35A08, 35D10, 35 B65.

The work is done while the author is a JSPS's fellow at the Institute of Mathematics, the University of Tsukuba, Japan.

Recelved May 25, 1999. 
We denote by $C$ a general constant which may vary from place to place. For two complex numbers $z_{1}, z_{2} \in C$ we define $z_{1}^{z_{2}}$ as $e^{z_{2} \ln z_{1}}$ and if $z_{1}=r e^{l \varphi},-\pi<$ $\varphi \leq \pi$ then $\ln z_{1}=\ln r+i \varphi$. Now let us recall the following lemma from [8].

Lemma 1. Assume that $\omega_{1}, \omega_{3} \in C, \operatorname{Re} \omega_{3}>-1$. Then we have

$$
\int_{0}^{\pi}(\sin \theta+i \cos \theta)^{\omega_{1}} \sin ^{\omega_{3}} \theta d \theta=\frac{2^{-\omega_{3}} \pi \Gamma\left(\omega_{3}+1\right)}{\Gamma\left(1+\left(\left(\omega_{3}-\omega_{1}\right) / 2\right)\right) \Gamma\left(1+\left(\left(\omega_{3}+\omega_{1}\right) / 2\right)\right)} .
$$

Proof. We refer to the proof in [8].

Lemma 2. Assume that $\omega_{1}, \omega_{2}, \omega_{3} \in C, \operatorname{Re} w_{1}, \quad \operatorname{Re} w_{2}>0, \quad \operatorname{Re} \omega_{3}>-1$. Then

(1) $\int_{0}^{\pi}\left(w_{1} \sin \theta+i \cos \theta\right)^{\omega_{1}}\left(w_{2} \sin \theta-i \cos \theta\right)^{\omega_{2}} \sin ^{\omega_{3}} \theta d \theta$

$$
=\frac{\pi \Gamma\left(\omega_{3}+1\right) F_{2}\left(\omega_{3}+1,-\omega_{1},-\omega_{2}, 1+\left(\left(\omega_{3}-\omega_{1}+\omega_{2}\right) / 2\right), 1+\left(\left(\omega_{3}+\omega_{1}-\omega_{2}\right) / 2\right),\left(1-w_{1}\right) / 2,\left(1-w_{2}\right) / 2\right)}{2^{\omega_{3}} \Gamma\left(1+\left(\left(\omega_{3}+\omega_{1}-\omega_{2}\right) / 2\right)\right) \Gamma\left(1+\left(\left(\omega_{3}-\omega_{1}+\omega_{2}\right) / 2\right)\right)},
$$

(2) $\int_{0}^{\pi}\left(w_{1} \sin \theta+i \cos \theta\right)^{\omega_{1}}\left(w_{2} \sin \theta+i \cos \theta\right)^{\omega_{2}} \sin ^{\omega_{3}} \theta d \theta$

$$
=\frac{\pi \Gamma\left(\omega_{3}+1\right) F_{1}\left(\omega_{3}+1,-\omega_{1},-\omega_{2}, 1+\left(\left(\omega_{3}-\omega_{1}-\omega_{2}\right) / 2\right),\left(1-w_{1}\right) / 2,\left(1-w_{2}\right) / 2\right)}{2^{\omega_{3}} \Gamma\left(1+\left(\left(\omega_{3}+\omega_{1}+\omega_{2}\right) / 2\right)\right) \Gamma\left(1+\left(\left(\omega_{3}-\omega_{1}-\omega_{2}\right) / 2\right)\right)},
$$

where $F_{1}\left(\alpha, \beta, \beta^{\prime}, \gamma, x, y\right), F_{2}\left(\alpha, \beta, \beta^{\prime}, \gamma, \gamma^{\prime}, x, y\right)$ are the first and second two-variable hypergeometric functions of Appel [10] defined as

$$
\begin{gathered}
F_{1}\left(\alpha, \beta, \beta^{\prime}, \gamma, x, y\right)=\sum \frac{(\alpha, m+n)(\beta, m)\left(\beta^{\prime}, n\right)}{(\gamma, m+n)(1, m)(1, n)} x^{m} y^{n}, \\
F_{2}\left(\alpha, \beta, \beta^{\prime}, \gamma, \gamma^{\prime}, x, y\right)=\sum \frac{(\alpha, m+n)(\beta, m)\left(\beta^{\prime}, n\right)}{(\gamma, m)\left(\gamma^{\prime}, n\right)(1, m)(1, n)} x^{m} y^{n} .
\end{gathered}
$$

Proof. Define the left side of (1), (2) by $F\left(\omega_{1}, \omega_{2}, \omega_{3}, w_{1}, w_{2}\right)$, $G\left(\omega_{1}, \omega_{2}, \omega_{3}, w_{1}, w_{2}\right)$. It is clear that $F, G$ are analytic functions of $\left(w_{1}, w_{2}\right)$ when $\operatorname{Re} w_{1}, \operatorname{Re} w_{2}>0$. First we prove the formula (1). We have

$$
\begin{aligned}
\frac{\partial^{m+n} F\left(\omega_{1}, \omega_{2}, \omega_{3}, w_{1}, w_{2}\right)}{\partial w_{1}^{m} \partial w_{2}^{n}} \\
=(-1)^{m+n} \times \int_{0}^{\pi}\left(-\omega_{1}, m\right)\left(-\omega_{2}, n\right)\left(w_{1} \sin \theta+i \cos \theta\right)^{\omega_{1}-m} \\
\quad \times\left(w_{2} \sin \theta-i \cos \theta\right)^{\omega_{2}-n} \sin ^{\omega_{3}+m+n} \theta d \theta \\
=(-1)^{m+n}\left(-\omega_{1}, m\right)\left(-\omega_{2}, n\right) F\left(\omega_{1}-m, \omega_{2}-n, \omega_{3}+m+n, w_{1}, w_{2}\right) .
\end{aligned}
$$


By using Lemma 1 we deduce that

$$
\begin{aligned}
(-1)^{m+n} & \left.\frac{\partial^{m+n} F\left(\omega_{1}, \omega_{2}, \omega_{3}, w_{1}, w_{2}\right)}{\partial w_{1}^{m} \partial w_{2}^{n}}\right|_{\left(w_{1}, w_{2}\right)=(1,1)} \\
& =\left(-\omega_{1}, m\right)\left(-\omega_{2}, n\right) F\left(\omega_{1}-m, \omega_{2}-n, \omega_{3}+m+n, 1,1\right) \\
& =\left(-\omega_{1}, m\right)\left(-\omega_{2}, n\right) \int_{0}^{\pi}(\sin \theta+i \cos \theta)^{\omega_{1}-m}(\sin \theta-i \cos \theta)^{\omega_{2}-n} \sin ^{\omega_{3}+m+n} \theta d \theta \\
& =\int_{0}^{\pi}\left(-\omega_{1}, m\right)\left(-\omega_{2}, n\right)(\sin \theta+i \cos \theta)^{\omega_{1}-m-\omega_{2}+n} \sin ^{\omega_{3}+m+n} \theta d \theta \\
& =\frac{\pi 2^{-\left(\omega_{3}+m+n\right)}\left(-\omega_{1}, m\right)\left(-\omega_{2}, n\right) \Gamma\left(\omega_{3}+1+m+n\right)}{\Gamma\left(1+n+\left(\left(\omega_{3}+\omega_{1}-\omega_{2}\right) / 2\right)\right) \Gamma\left(1+m+\left(\left(\omega_{3}-\omega_{1}+\omega_{2}\right) / 2\right)\right)} .
\end{aligned}
$$

Hence the desired formula follows.

Now we proceed to prove the formula (2). We can repeat the above proof with $F$ replaced by $G$. The only difference is

$$
\begin{aligned}
\left(-\omega_{1}, m\right)\left(-\omega_{2}, n\right) G\left(\omega_{1}-m, \omega_{2}-n, \omega_{3}+m+n, 1,1\right) \\
\quad=\left(-\omega_{1}, m\right)\left(-\omega_{2}, n\right) \int_{0}^{\pi}(\sin \theta+i \cos \theta)^{\omega_{1}-m}(\sin \theta+i \cos \theta)^{\omega_{2}-n} \sin ^{\omega_{3}+m+n} \theta d \theta \\
\quad=\int_{0}^{\pi}\left(-\omega_{1}, m\right)\left(-\omega_{2}, n\right)(\sin \theta+i \cos \theta)^{\omega_{1}-m+\omega_{2}-n} \sin ^{\omega_{3}+m+n} \theta d \theta \\
\quad=\frac{\pi 2^{-\left(\omega_{3}+m+n\right)}\left(-\omega_{1}, m\right)\left(-\omega_{2}, n\right) \Gamma\left(\omega_{3}+1+m+n\right)}{\Gamma\left(1+\left(\left(\omega_{3}+\omega_{1}+\omega_{2}\right) / 2\right)\right) \Gamma\left(1+m+n+\left(\left(\omega_{3}-\omega_{1}-\omega_{2}\right) / 2\right)\right)} .
\end{aligned}
$$

Hence the desired formula follows.

COROLLARY 1. Under the assumptions of Lemma 2, if moreover $\omega_{1}+\omega_{2}+$ $\omega_{3}=-2$, then

$$
\begin{aligned}
& \int_{0}^{\pi}\left(w_{1} \sin \theta+i \cos \theta\right)^{\omega_{1}}\left(w_{2} \sin \theta-i \cos \theta\right)^{\omega_{2}} \sin ^{\omega_{3}} \theta d \theta \\
& \quad=\frac{2 \pi \Gamma\left(\omega_{3}+1\right)}{\left(w_{1}+w_{2}\right)^{\omega_{3}+1} \Gamma\left(1+\left(\left(\omega_{3}+\omega_{1}-\omega_{2}\right) / 2\right)\right) \Gamma\left(1+\left(\left(\omega_{3}-\omega_{1}+\omega_{2}\right) / 2\right)\right)} .
\end{aligned}
$$

Proof. By Lemma 2 we have

$$
\begin{aligned}
& \int_{0}^{\pi}\left(w_{1} \sin \theta+i \cos \theta\right)^{\omega_{1}}\left(w_{2} \sin \theta-i \cos \theta\right)^{\omega_{2}} \sin ^{\omega_{3}} \theta d \theta \\
& \quad=\frac{\pi \Gamma\left(\omega_{3}+1\right) F_{2}\left(1+\omega_{3},-\omega_{1},-\omega_{2},-\omega_{1},-\omega_{2},\left(1-w_{1}\right) / 2,\left(1-w_{2}\right) / 2\right)}{2^{\omega_{3}} \Gamma\left(1+\left(\left(\omega_{3}+\omega_{1}-\omega_{2}\right) / 2\right)\right) \Gamma\left(1+\left(\left(\omega_{3}-\omega_{1}+\omega_{2}\right) / 2\right)\right)} .
\end{aligned}
$$


Now using the following relation (see $[10$, p. 15])

$$
F_{2}\left(\alpha, \beta, \beta^{\prime}, \beta, \beta^{\prime}, x, y\right)=(1-x-y)^{-\alpha}
$$

gives the desired result.

COROLlaRY 2. Under the assumptions of Lemma 2, if moreover $\omega_{1}+\omega_{2}+$ $\omega_{3}$ or $-\omega_{1}-\omega_{2}+\omega_{3}$ is some even negative integer then

$$
\int_{0}^{\pi}\left(w_{1} \sin \theta+i \cos \theta\right)^{\omega_{1}}\left(w_{2} \sin \theta+i \cos \theta\right)^{\omega_{2}} \sin ^{\omega_{3}} \theta d \theta=0 .
$$

Proof. Indeed, in that case $1+\left(\left(\omega_{3}+\omega_{1}+\omega_{2}\right) / 2\right)$ or $1+$ $\left(\left(\omega_{3}-\omega_{1}-\omega_{2}\right) / 2\right)$ will be a pole of the function $\Gamma($.$) .$

\section{§3. Main results}

In the case $\operatorname{Re} a<0$, and $\operatorname{Re} b>0$ we will consider a function of the following form

$$
F_{k, a, b}^{\alpha, \beta, \gamma}(x, y)=\left(b x^{k+1}-i(k+1) y\right)^{\alpha}\left(-a x^{k+1}+i(k+1) y\right)^{\beta} x^{\gamma} .
$$

If $\operatorname{Re} a<0, \operatorname{Re} b<0$ and $a \neq b$ then we will consider the following function

$$
E_{k, a, b}^{\alpha, \beta, \gamma}(x, y)=\left(-b x^{k+1}+i(k+1) y\right)^{\alpha}\left(-a x^{k+1}+i(k+1) y\right)^{\beta} x^{\gamma} .
$$

In the resonance case $a=b, \operatorname{Re} a<0$ we consider the following function

$$
R_{k, a}^{\kappa, \eta, \gamma}(x, y)=x^{\gamma}\left(-a x^{k+1}+i(k+1) y\right)^{\eta} e^{\kappa x^{k+1} /\left(-a x^{k+1}+i(k+1) y\right)} .
$$

It is clear that the differentiating of $F_{k, a, b}^{\alpha, \beta, \gamma}(x, y)$ and $E_{k, a, b}^{\alpha, \beta, \gamma}(x, y)$ formally differ from each other by a factor -1 . Note that

$$
\begin{aligned}
& X_{1}\left(-a x^{k+1}+i(k+1) y\right)=(k+1)(b-a) x^{k}, X_{1}\left(b x^{k+1}-i(k+1) y\right)=0, \\
& X_{2}\left(-a x^{k+1}+i(k+1) y\right)=0, X_{2}\left(b x^{k+1}-i(k+1) y\right)=(k+1)(b-a) x^{k} .
\end{aligned}
$$

Therefore we have

$$
\begin{aligned}
G_{k, c}^{a, b} F_{k, a, b}^{\alpha, \beta, \gamma}(x, y)= & \left(b x^{k+1}-i(k+1) y\right)^{\alpha-1}\left(-a x^{k+1}+i(k+1) y\right)^{\beta-1} x^{\gamma-2} \\
& \times\left\{\left[(k+1)^{2}(b-a)^{2} \alpha \beta+(k+1)(k+\gamma)(b-a) b \beta\right.\right. \\
& -(k+1) a(b-a) \alpha \gamma-\gamma(\gamma-1) a b \\
& -(k+1) c(a \alpha+b \beta)] x^{2 k+2}+(k+1)^{2} \gamma(\gamma-1) y^{2} \\
& +i\left[-(k+1)^{2}(k+\gamma)(b-a) \beta\right. \\
& +(k+1)^{2} c(\alpha+\beta)+(k+1)^{2}(b-a) \alpha \gamma \\
& \left.+(k+1) \gamma(\gamma-1)(a+b)] x^{k+1} y\right\} .
\end{aligned}
$$


Hence in the non-resonance case $a \neq b$ we formally have $G_{k, c}^{a, b} F_{k, a, b}^{\alpha, \beta, \gamma}(x, y)=$ $-G_{k, c}^{a, b} E_{k, a, b}^{\alpha, \beta, \gamma}(x, y)=0$ if

$\gamma=0, \alpha=0, \beta=0$, the solution is a constant.

$\gamma=0, c=k(b-a), \alpha=0, \beta$ arbitrary $\neq 0$.

$\gamma=0, c=0, \alpha$ arbitrary $\neq 0, \beta=0$.

$\gamma=0, \alpha=(c-k(b-a)) /((k+1)(b-a))=: \alpha_{1}, \beta=-c /((k+1)(b-a))=: \beta_{1}$.

$\gamma=1, \alpha=0, \beta=0$, the solution is the linear function $x$.

$\gamma=1, c=(b-a)(k+1), \alpha=0, \beta$ arbitrary $\neq 0$.

$\gamma=1, c=-(b-a), \alpha$ arbitrary $\neq 0, \beta=0$.

$\gamma=1, \quad \alpha=(c-(k+1)(b-a)) /((k+1)(b-a))=: \alpha_{2}, \quad \beta=-(c+(b-a)) /$ $((k+1)(b-a))=: \beta_{2}$.

In the resonance case $a=b$ we can consider $R_{k, a}^{\kappa, \eta, \gamma}(x, y)$ as the limit of $E_{k, a, b}^{\alpha_{1}, \beta_{1}, 0}(x, y), E_{k, a, b}^{\alpha_{2}, \beta_{2}, 1}(x, y)$ when $b \rightarrow a$. Thus we will have

$$
\begin{gathered}
\gamma=0, \eta=-\frac{k}{k+1}=: \eta_{1}, \quad \kappa=-\frac{c}{k+1}=: \kappa_{1} \\
\text { or } \gamma=1, \eta=-\frac{k+2}{k+1}=: \eta_{2}, \quad \kappa=-\frac{c}{k+1}=: \kappa_{2},
\end{gathered}
$$

and the formal equations $G_{k, c}^{a, a} R_{k, a}^{\kappa_{1}, \eta_{1}, 0}(x, y)=0, G_{k, c}^{a, a} R_{k, a}^{\kappa_{2}, \eta_{2}, 1}(x, y)=0$.

THEOREM 1. Assume that $k$ is odd. If $\operatorname{Re} a<0$, and $\operatorname{Re} b>0$ then

I) $G_{k, c}^{a, b} F_{k, a, b}^{\alpha_{1}, \beta_{1}, 0}(x, y)$

$$
=-\frac{4(b-a)^{1 /(k+1)} \pi \Gamma(k /(k+1))}{\Gamma((k(b-a)-c) /((k+1)(b-a))) \Gamma(c /((k+1)(b-a)))} \delta(x, y)=: A_{k, c}^{a, b} \delta(x, y) .
$$

II) $G_{k, k(b-a)}^{a, b} F_{k, a, b}^{0, \beta, 0}(x, y)=0$ if $\operatorname{Re} \beta>-k /(k+1)$.

III) $G_{k, 0}^{a, b} F_{k, a, b}^{\alpha, 0,0}(x, y)=0$ if $\operatorname{Re} \alpha>-k /(k+1)$.

If $\operatorname{Re} a<0, \operatorname{Re} b<0$ and $a \neq b$ then

IV) $G_{k, c}^{a, b} E_{k, a, b}^{\alpha_{1}, \beta_{1}, 0}(x, y)=0$.

If $\operatorname{Re} a<0$ then

V) $G_{k, c}^{a, a} R_{k, a}^{\kappa_{1}, \eta_{1}, 0}(x, y)=0$.

Proof. I) We begin by noting that if $k$ is odd and $\operatorname{Re} a<0$, and $\operatorname{Re} b>0$ then $\left(b x^{k+1}-i(k+1) y\right)^{\alpha}$ and $\left(-a x^{k+1}+i(k+1) y\right)^{\beta} \in C^{\infty}\left(\boldsymbol{R}^{2} \backslash(0,0)\right)$ for every $\alpha$ and $\beta$. Let us introduce the following "polar coordinate"

$$
x=\rho(\sin \theta)_{ \pm}^{1 /(k+1)}, \quad y=\frac{\rho^{k+1}}{k+1} \cos \theta, \quad d x d y=\frac{\rho^{k+1}}{k+1}|\sin \theta|^{-k /(k+1)} d \rho d \theta .
$$

Here we use the following notation $(\sin \theta)_{ \pm}^{r}=\operatorname{sign}(\sin \theta)|\sin \theta|^{r}$ for every $r \in \boldsymbol{R}$. Note that the map $(x, y) \rightarrow(\rho, \theta)$ is not a differmorphism along the line $x=0$. 
But it is good enough for us because in the future we will use it only for integration, and if necessary we can take integrals as a limit. It is easy to verify that $\rho^{2 k+2}=x^{2 k+2}+(k+1)^{2} y^{2}$ Let us write $F_{k}^{1}(x, y)=F_{k, a, b}^{\alpha_{1}, \beta_{1}, 0}(x, y)$. First we prove that $F_{k}^{1}(x, y) \in L_{l o c}^{(k+2) / k)-\tau}\left(\boldsymbol{R}^{2}\right)$ for any small positive $\tau$. Indeed, since $F_{k}^{1}(x, y) \in C^{\infty}\left(\boldsymbol{R}^{2} \backslash(0,0)\right)$ it suffices to prove that $F_{k}^{1}(x, y) \in L^{((k+2) / k)-\tau}\left(B_{\varepsilon}\right)$, where $B_{\varepsilon}=\{(x, y) \mid \rho(x, y)<\varepsilon\}$. We have

$$
\begin{aligned}
\int_{B_{\varepsilon}}\left|F_{k}^{1}(x, y)\right|^{((k+2) / k)-\tau} d x d y & \leq C \int_{-\pi}^{\pi}|\sin \theta|^{-k /(k+1)} d \theta \int_{0}^{\varepsilon} \rho^{k+1}\left(\rho^{-k}\right)^{((k+2) / k)-\tau} d \rho \\
& \leq C \int_{0}^{\varepsilon} \rho^{-1+\tau k} d \rho<\infty .
\end{aligned}
$$

Note that $F_{k}^{1}(x, y) \notin L_{l o c}^{(k+2) / k}\left(\boldsymbol{R}^{2}\right) . \quad$ Let $\boldsymbol{R}_{\varepsilon}^{2}=\left\{(x, y) \in \boldsymbol{R}^{2} \mid \rho(x, y) \geq \varepsilon\right\} . \quad$ By applying Green's formula we have

$$
\begin{aligned}
\int_{\mathrm{R}_{\varepsilon}^{2}} f(x, y) G_{k,-c}^{b, a} v(x, y) d x d y \\
=\int_{\mathrm{R}_{\varepsilon}^{2}} v(x, y) G_{k, c}^{a, b} f(x, y) d x d y \\
\quad-\int_{\rho=\varepsilon} v(x, y)\left\{\left(v_{1}-i a x^{k} v_{2}\right) X_{1} f(x, y)+i c x^{k-1} v_{2} . f(x, y)\right\} d s \\
\quad+\int_{\rho=\varepsilon} f(x, y)\left(v_{1}-i b x^{k} v_{2}\right) X_{2} v d s=: \int_{\mathrm{R}_{\varepsilon}^{2}} V(f, v, k, a, b, c) d x d y \\
\quad-\int_{\rho=\varepsilon} v(x, y) B_{1}(f, k, a, b, c) d s+\int_{\rho=\varepsilon} f(x, y) B_{2}(v, k, a, b) d s
\end{aligned}
$$

for every $v(x, y) \in C_{0}^{\infty}\left(\boldsymbol{R}^{2}\right), f(x, y) \in C^{\infty}\left(\boldsymbol{R}^{2} \backslash(0,0)\right)$, where $v=\left(v_{1}, v_{2}\right)$ is the unit outward normal to $\partial \boldsymbol{R}_{\varepsilon}^{2}$. Replace $f(x, y)$ in (3) by $F_{k}^{1}(x, y)$ we obtain that

$$
\begin{aligned}
\int_{\mathrm{R}_{\varepsilon}^{2}} & F_{k}^{1}(x, y) G_{k,-c}^{b, a} v(x, y) d x d y \\
= & \int_{\mathrm{R}_{\varepsilon}^{2}} V\left(F_{k}^{1}, v, k, a, b, c\right) d x d y \\
& \quad-\int_{\rho=\varepsilon} v(x, y) B_{1}\left(F_{k}^{1}, k, a, b, c\right) d s+\int_{\rho=\varepsilon} F_{k}^{1}(x, y) B_{2}(v, k, a, b) d s .
\end{aligned}
$$

The first integral in the right side of (4) vanishes. We now compute the third integral in the right side of (4). It is easy to check that

$$
\left.d s\right|_{\partial B_{\varepsilon}}=\frac{1}{k+1}\left(\varepsilon^{2}|\sin \theta|^{-2 k /(k+1)} \cos ^{2} \theta+\varepsilon^{2 k+2} \sin ^{2} \theta\right)^{1 / 2} d \theta \quad \text { and }
$$




$$
\left.v\right|_{\partial B_{\varepsilon}}=\left.\left(v_{1}, v_{2}\right)\right|_{\partial B_{\varepsilon}}=-\left.\left(\frac{x^{2 k+1}}{\left(x^{4 k+2}+(k+1)^{2} y^{2}\right)^{1 / 2}}, \frac{(k+1) y}{\left(x^{4 k+2}+(k+1)^{2} y^{2}\right)^{1 / 2}}\right)\right|_{\partial B_{\varepsilon}} .
$$

Hence

$$
\left.\left(x^{4 k+2}+(k+1)^{2} y^{2}\right)^{-1 / 2} d s\right|_{\partial B_{\varepsilon}}=\frac{1}{k+1} \varepsilon^{-k}|\sin \theta|^{-k /(k+1)} d \theta .
$$

It follows that

$$
\begin{aligned}
\left|\int_{\rho=\varepsilon} F_{k}^{1}(x, y) B_{2}(v, k, a, b) d s\right| & \leq C \int_{\rho=\varepsilon}\left|F_{k}^{1}(x, y)\right| .\left(\left|v_{1}\right|+\left|v_{2} \cdot x^{k}\right|\right) d s \\
& \leq C \varepsilon \int_{-\pi}^{\pi}(|\sin \theta|+|\cos \theta|) d \theta \rightarrow 0 \quad \text { as } \varepsilon \rightarrow 0
\end{aligned}
$$

Next we evaluate $B_{1}\left(F_{k}^{1}, k, a, b, c\right)$. We have

$$
\begin{aligned}
B_{1}\left(F_{k}^{1}, k, a, b, c\right)= & c x^{k-1}\left(b x^{k+1}-i(k+1) y\right)^{\alpha_{1}}\left(-a x^{k+1}+i(k+1) y\right)^{\beta_{1}-1} \\
& \times\left(x^{2 k+2}+(k+1)^{2} y^{2}\right)\left(x^{4 k+2}+(k+1)^{2} y^{2}\right)^{-1 / 2} .
\end{aligned}
$$

Therefore applying Corollary 1 with $\omega_{1}=\beta_{1}-1=-(c+(k+1)(b-a)) /$ $((k+1)(b-a)), \quad \omega_{2}=\alpha_{1}=(c-k(b-a)) /((k+1)(b-a)), \quad \omega_{3}=-(1 /(k+1))$, $w_{1}=-a, w_{2}=b$ we obtain

$$
\begin{aligned}
& -\int_{\rho=\varepsilon} v(x, y) B_{1}\left(F_{k}^{1}, k, a, b, c\right) d s \\
& =-\frac{c}{k+1} \int_{-\pi}^{\pi}|\sin \theta|^{-1 /(k+1)}(-a|\sin \theta|+i \cos \theta)^{\beta_{1}-1} \\
& \quad \times(b|\sin \theta|-i \cos \theta)^{\alpha_{1}} v(\varepsilon, \theta) d \theta \\
& =-\frac{c}{k+1} \int_{-\pi}^{\pi}(v(0,0)+\bar{o}(1))|\sin \theta|^{-1 /(k+1)} \\
& \quad \times(-a|\sin \theta|+i \cos \theta)^{\beta_{1}-1}(b|\sin \theta|-i \cos \theta)^{\alpha_{1}} d \theta \\
& =-\frac{2 v(0,0) c}{k+1} \int_{0}^{\pi} \sin ^{-1 /(k+1)} \theta(-a \sin \theta+i \cos \theta)^{\beta_{1}-1} \\
& \quad \times(b \sin \theta-i \cos \theta)^{\alpha_{1}} d \theta+\bar{o}(1) \\
& =-\frac{4(b-a)^{1 /(k+1)} \pi \Gamma(k /(k+1))}{\Gamma((k(b-a)-c) /((k+1)(b-a))) \Gamma(c /((k+1)(b-a)))} v(0,0)+\bar{o}(1) .
\end{aligned}
$$

Now from (4), (5), (6) we deduce that 


$$
\begin{aligned}
\left(G_{k, c}^{a, b} F_{k}^{1}(x, y), v(x, y)\right) & =\left(F_{k}^{1}(x, y), G_{k,-c}^{a, b} v(x, y)\right) \\
& =\lim _{\varepsilon \rightarrow 0} \int_{\rho \geq \varepsilon} F_{k}^{1}(x, y) G_{k,-c}^{a, b} v(x, y) d x d y \\
& =-\frac{4(b-a)^{1 /(k+1)} \pi \Gamma(k /(k+1))}{\Gamma((k(b-a)-c) /((k+1)(b-a))) \Gamma(c /((k+1)(b-a)))} v(0,0) .
\end{aligned}
$$

Hence $G_{k, c}^{a, b} F_{k, a, b}^{\alpha_{1}, \beta_{1}, 0}(x, y)=A_{k, c}^{a, b} \delta(x, y)$.

II) Assume that $\operatorname{Re} \beta>-k /(k+1)$. We then have $F_{k, a, b}^{0, \beta, 0}(x, y)=$ $\left(-a x^{k+1}+i(k+1) y\right)^{\beta} \in L_{l o c}^{p}\left(\boldsymbol{R}^{2}\right)$ for every $1 \leq p<-(k+2) /((k+1) \operatorname{Re} \beta)$ if $\operatorname{Re} \beta<0$ and $F_{k, a, b}^{0, \beta, 0}(x, y) \in L_{l o c}^{\infty}\left(\boldsymbol{R}^{2}\right)$ if $\operatorname{Re} \beta \geq 0$. It is easy to compute that

$$
\begin{aligned}
&\left.B_{1}\left(F_{k, a, b}^{0, \beta, 0}, k, a, b, k(b-a)\right)\right|_{\rho=\varepsilon} \\
&=(k+1)(b-a) x^{k-1}\left(-a x^{k+1}+i(k+1) y\right)^{\beta-1} \\
& \times\left\{-\beta x^{2 k+2}+i a(k+(k+1) \beta) x^{k+1} y+k(k+1) y^{2}\right\} \\
& \times\left.\left(x^{4 k+2}+(k+1)^{2} y^{2}\right)^{-1 / 2}\right|_{\rho=\varepsilon} \\
&= \varepsilon^{2 k+(k+1) \beta}(b-a)\left(-\beta(k+1) \sin ^{2} \theta+i a(k+k \beta+\beta) \cos \theta|\sin \theta|+k \cos ^{2} \theta\right) \\
& \times|\sin \theta|^{(k-1) /(k+1)}(-a|\sin \theta|+i \cos \theta)^{\beta-1} \\
& \times\left(\varepsilon^{4 k+2}|\sin \theta|^{(4 k+2) /(k+1)}+\varepsilon^{2 k+2} \cos ^{2} \theta\right)^{-1 / 2}
\end{aligned}
$$

and

$$
\left.\left|\left(\left|v_{1}\right|+\left|v_{2} . x^{k}\right|\right) F_{k, a, b}^{0, \beta, 0}\right| d s\right|_{\rho=\varepsilon} \leq C \varepsilon^{(k+1)(1+\operatorname{Re} \beta)}\left|(-a|\sin \theta|+i \cos \theta)^{\beta}\right| d \theta .
$$

Using the assumption that $\operatorname{Re} \beta>-k /(k+1)$ we deduce that

$$
-\int_{\rho=\varepsilon} v(x, y) B_{1}\left(F_{k, a, b}^{0, \beta, 0}, k, a, b, k(b-a)\right) d s+\int_{\rho=\varepsilon} F_{k, a, b}^{0, \beta, 0}(x, y) B_{2}(v, k, a, b) d s \rightarrow 0
$$

as $\varepsilon \rightarrow 0$. Hence $G_{k, k(b-a)}^{a, b} F_{k, a, b}^{0, \beta, 0}(x, y)=0$.

III) The proof of this part is the same as the proof of part II) with $-a, \beta$ replaced by $b, \alpha$.

IV) We note that if $k$ is odd and $\operatorname{Re} a<0$, and $\operatorname{Re} b<0$ then $\left(-b x^{k+1}+i(k+1) y\right)^{\alpha}$ and $\left(-a x^{k+1}+i(k+1) y\right)^{\beta} \in C^{\infty}\left(\boldsymbol{R}^{2} \backslash(0,0)\right)$ for every $\alpha$ and $\beta$. Therefore we can repeat all the arguments in part I). The only difference is

$$
\begin{aligned}
& -\int_{\rho=\varepsilon} v(x, y) B_{1}\left(E_{k, a, b}^{\alpha_{1}, \beta_{1}, 0}, k, a, b, c\right) d s+\int_{\rho=\varepsilon} E_{k, a, b}^{\alpha_{1}, \beta_{1}, 0}(x, y) B_{2}(v, k, a, b) d s \\
& =-\frac{2 v(0,0) c}{k+1} \int_{0}^{\pi} \sin ^{-1 /(k+1)} \theta(-a \sin \theta+i \cos \theta)^{\beta_{1}-1}(-b \sin \theta-i \cos \theta)^{\alpha_{1}} d \theta+\bar{o}(1) .
\end{aligned}
$$


Applying Corollary 2 with $\omega_{1}=\beta_{1}-1=-(c+(k+1)(b-a)) /((k+1)(b-a))$, $\omega_{2}=\alpha_{1}=(c-k(b-a)) /((k+1)(b-a)), \omega_{3}=-1 /(k+1), w_{1}=-a, w_{2}=-b$ we obtain

(7) $-\frac{2 v(0,0) c}{k+1} \int_{0}^{\pi} \sin ^{-1 /(k+1)} \theta(-a \sin \theta+\imath \cos \theta)^{\beta_{1}-1}(-b \sin \theta+i \cos \theta)^{\alpha_{1}} d \theta=0$.

Now clearly we have $G_{k, c}^{a, b} E_{k, a, b}^{\alpha_{1}, \beta_{1}, 0}(x, y)=0$.

V) Argue as in part I) we arrive at

$$
\begin{aligned}
-\int_{\rho=\varepsilon} & v(x, y) B_{1}\left(R_{k, a}^{\kappa_{1}, \eta_{1}, 0}, k, a, a, c\right) d s+\int_{\rho=\varepsilon} R_{k, a}^{\kappa_{1}, \eta_{1}, 0}(x, y) B_{2}(v, k, a, a) d s \\
= & -\frac{2 v(0,0) c}{k+1} \int_{0}^{\pi} \sin ^{-1 /(k+1)} \theta(-a \sin \theta+i \cos \theta)^{\eta_{1}-1} \\
& \times e^{\kappa_{1} \sin \theta /(-a \sin \theta+\imath \cos \theta)} d \theta+\bar{o}(1)=\bar{o}(1) .
\end{aligned}
$$

The last equality is a consequence of (7) when we let $b \rightarrow a$. Therefore we conclude that $G_{k, c}^{a, a} R_{k, a}^{\kappa_{1}, \eta_{1}, 0}(x, y)=0$. This concludes the proof of Theorem 1.

COROLlaRY 3. If either $\operatorname{Re} a<0, \operatorname{Re} b>0, c=-(k+1)(b-a) N$, or $\operatorname{Re} a<0, \operatorname{Re} b>0, c=((k+1) N+k)(b-a)$, where $N$ is a non-negative integer, or $\operatorname{Re} a<0, \operatorname{Re} b<0$, then $G_{k, c}^{a, b}$ is not hypoelliptic (nor analytic hypoelliptic).

Proof. Indeed, if $\operatorname{Re} a<0, \operatorname{Re} b>0, c=-(k+1)(b-a) N$, or $\operatorname{Re} a<0$, $\operatorname{Re} b>0, c=((k+1) N+k)(b-a)$ then $\Gamma((k(b-a)-c) /((k+1)(b-a)))=\infty$ or $\Gamma(c /((k+1)(b-a)))=\infty \Rightarrow A_{k, c}^{a, b}=0 \Rightarrow G_{k, c}^{a, b} F_{k, a, b}^{\alpha_{1}, \beta_{1}, 0}(x, y)=0$. If $\operatorname{Re} a<0$, $\operatorname{Re} b<0$, then $G_{k, c}^{a, b} E_{k, a, b}^{\alpha_{1}, \beta_{1}, 0}(x, y)=0$ in the non-resonance case or $G_{k, c}^{a, a} R_{k, a}^{\kappa_{1}, \eta_{1}, 0}(x, y)=0$ in the resonance case.

THEOREM 2. Assume that $k$ is odd. If $\operatorname{Re} a<0$, and $\operatorname{Re} b>0$ then I) $G_{k, c}^{a, b} F_{k, a, b}^{\alpha_{2}, \beta_{2}, 1}(x, y)$

$$
\begin{aligned}
= & \frac{4 \pi \Gamma((k+2) /(k+1))}{(b-a)^{1 /(k+1)} \Gamma(((k+1)(b-a)-c) /((k+1)(b-a))) \Gamma((c+b-a) /((k+1)(b-a)))} \\
& \cdot \frac{\partial \delta(x, y)}{\partial x}=: B_{k, c}^{a, b} \frac{\partial \delta(x, y)}{\partial x} .
\end{aligned}
$$

II) $G_{k,(k+1)(b-a)}^{a, b} F_{k, a, b}^{0, \beta, 1}(x, y)=0$ if $\operatorname{Re} \beta>-(k+2) /(k+1)$.

III) $G_{k,-(b-a)}^{a, b} F_{k, a, b}^{\alpha, 0,1}(x, y)=0$ if $\operatorname{Re} \alpha>-(k+2) /(k+1)$.

If $\operatorname{Re} a<0, \operatorname{Re} b<0$ and $a \neq b$ then

IV) $G_{k, c}^{a, b} E_{k, a, b}^{\alpha_{2}, \beta_{2}, 1}(x, y)=0$.

If $\operatorname{Re} a<0$ then

V) $G_{k, c}^{a, a} R_{k, a}^{\kappa_{2}, \eta_{2}, 1}(x, y)=0$. 
Proof. I) Let us write $F_{k}^{2}(x, y)=F_{k, a, b}^{\alpha_{2}, \beta_{2}, 1}(x, y)$. As in the proof of Theorem 1 it is easy to check that $F_{k}^{2}(x, y) \in L_{l o c}^{((k+2) /(k+1))-\tau}\left(\boldsymbol{R}^{2}\right)$ for any small positive $\tau$. We see that

$$
\begin{gathered}
\mid \int_{\rho=\varepsilon} a x^{k}\left(v_{1}-i v_{2} b x^{k} \cdot F_{k}^{2}(x, y) \cdot \frac{\partial v(x, y)}{\partial y} d s \mid\right. \\
\quad \leq C \varepsilon \int_{-\pi}^{\pi}\left|\frac{\partial v(x, y)}{\partial y}\right| d \theta \rightarrow 0 \text { as } \varepsilon \rightarrow 0 .
\end{gathered}
$$

Next we have

$$
\begin{aligned}
\int_{\rho=\varepsilon} & F_{k}^{2}(x, y) \cdot\left(v_{1}-i b x^{k} v_{2}\right) \cdot \frac{\partial v(x, y)}{\partial x} d s \\
= & \frac{1}{k+1} \int_{-\pi}^{\pi} \frac{\partial v(\varepsilon, \theta)}{\partial x} \sin ^{1 /(k+1)} \theta(-a|\sin \theta|+i \cos \theta)^{\beta_{2}-1}(b|\sin \theta|-i \cos \theta)^{\alpha_{2}} \\
& \times\left(a \sin ^{2} \theta-i(a b+1)|\sin \theta| \cos \theta-b \cos ^{2} \theta\right) d \theta \\
= & \frac{2(\partial v(0,0) / \partial x)}{k+1} \int_{0}^{\pi} \sin ^{1 /(k+1)} \theta(-a|\sin \theta|+i \cos \theta)^{\beta_{2}-1}(b|\sin \theta|-i \cos \theta)^{\alpha_{2}} \\
& \quad \times\left(a \sin ^{2} \theta-i(a b+1)|\sin \theta| \cos \theta-b \cos ^{2} \theta\right) d \theta+\bar{o}(1) .
\end{aligned}
$$

Now let us compute $B_{1}\left(F_{k}^{2}, k, a, b, c\right)$. We have

$$
\begin{aligned}
B_{1}\left(F_{k}^{2}, k\right. & , a, b, c)\left.\right|_{\rho=\varepsilon} \\
= & x^{k}\left(-a x^{k+1}+i(k+1) y\right)^{\beta_{2}-1}\left(b x^{k+1}-i(k+1) y\right)^{\alpha_{2}} \\
& \times\left((c+b) x^{2 k+2}-i(k+1)(a b+1) y x^{k+1}+(k+1)^{2}(c-a) y^{2}\right) \\
& \times\left.\left(x^{4 k+2}+(k+1)^{2} y^{2}\right)^{-1 / 2}\right|_{\rho=\varepsilon}=\varepsilon^{k-1}(\sin \theta)_{ \pm}^{k /(k+1)}(-a|\sin \theta|+i \cos \theta)^{\beta_{2}-1} \\
& \times(b|\sin \theta|-i \cos \theta)^{\alpha_{2}}\left(\varepsilon^{4 k+2}|\sin \theta|^{(4 k+2) /(k+1)}+\varepsilon^{2 k+2} \cos ^{2} \theta\right)^{-1 / 2} \\
& \times\left((c+b) \sin ^{2} \theta-i(a b+1)|\sin \theta| \cos \theta+(c-a) \cos ^{2} \theta\right) .
\end{aligned}
$$

Note that $v(\varepsilon, \theta)=v(0,0)+\varepsilon(\sin \theta)_{ \pm}^{1 /(k+1)}(\partial v(0,0) / \partial x)+\bar{o}(\varepsilon)$. It follows that

$$
\begin{aligned}
-\int_{\rho=\varepsilon} v(x, y) B_{1}\left(F_{k}^{2}, k, a, b, c\right) d s \\
=-\frac{v(0,0)}{(k+1) \varepsilon} \int_{-\pi}^{\pi} \operatorname{sign}(\sin \theta)(-a|\sin \theta|+i \cos \theta)^{\beta_{2}-1}(b|\sin \theta|-i \cos \theta)^{\alpha_{2}} \\
\quad \times\left((c+b) \sin ^{2} \theta-i(a b+1)|\sin \theta| \cos \theta+(c-a) \cos ^{2} \theta\right) d \theta
\end{aligned}
$$




$$
\begin{aligned}
& -\frac{2(\partial v(0,0) / \partial x)}{k+1} \int_{0}^{\pi} \sin ^{1 /(k+1)} \theta \\
& \quad \times(-a|\sin \theta|+i \cos \theta)^{\beta_{2}-1}(b|\sin \theta|-i \cos \theta)^{\alpha_{2}} \\
& \quad \times\left((c+b) \sin ^{2} \theta-i(a b+1)|\sin \theta| \cos \theta+(c-a) \cos ^{2} \theta\right) d \theta+\bar{o}(1) .
\end{aligned}
$$

We see that the first integral in the right side of (10) vanishes since the integrand is an odd function of $\theta$. Therefore summing (8), (9), and (10) and applying Corollary 1 with $\omega_{1}=\beta_{2}-1=-(c+(k+2)(b-a)) /((k+1)(b-a)), \omega_{2}=\alpha_{2}$ $=(c-(k+1)(b-a)) /((k+1)(b-a)), \quad \omega_{3}=1 /(k+1), \quad w_{1}=-a, \quad w_{2}=b$ yields

$$
\begin{aligned}
(11) & -\int_{\rho=\varepsilon} v(x, y) B_{1}\left(F_{k}^{2}, k, a, b, c\right) d s+\int_{\rho=\varepsilon} F_{2}^{k}(x, y) B_{2}(v, k, a, b) d s \\
= & -\frac{2(c+b-a)(\partial v(0,0) / \partial x)}{k+1} \\
& \times \int_{0}^{\pi} \sin ^{1 /(k+1)} \theta(-a|\sin \theta|+i \cos \theta)^{\beta_{2}-1}(b|\sin \theta|-i \cos \theta)^{\alpha_{2}} d \theta+\bar{o}(1) \\
= & -\frac{4 \pi \Gamma((k+2) /(k+1))}{(b-a)^{1 /(k+1)} \Gamma(((k+1)(b-a)-c) /((k+1)(b-a))) \Gamma((c+b-a) /((k+1)(b-a)))} \\
& \times \frac{\partial v(0,0)}{\partial x}+\bar{o}(1) .
\end{aligned}
$$

By (4) with $F_{k}^{1}(x, y)$ replaced by $F_{k}^{2}(x, y)$ and (11) we deduce that

$$
\begin{aligned}
& \left(G_{k, c}^{a, b} F_{k}^{2}(x, y), v(x, y)\right) \\
& =\left(F_{k}^{2}(x, y), G_{k,-c}^{a, b} v(x, y)\right) \\
& =\lim _{\varepsilon \rightarrow 0} \int_{\rho \geq \varepsilon} F_{k}^{2}(x, y) G_{k,-c}^{a, b} v(x, y) d x d y \\
& =-\frac{4 \pi \Gamma((k+2) /(k+1))(\partial v(0,0) / \partial x)}{(b-a)^{1 /(k+1)} \Gamma(((k+1)(b-a)-c) /((k+1)(b-a))) \Gamma((c+b-a) /((k+1)(b-a)))} .
\end{aligned}
$$

It follows that $G_{k, c}^{a, b} F_{k, a, b}^{\alpha_{2}, \beta_{2}, 1}(x, y)=B_{k, c}^{a, b}(\partial \delta(x, y) / \partial x)$.

II) Assume that $\operatorname{Re} \beta>-(k+2) /(k+1)$. We then have $F_{k, a, b}^{0, \beta, 1}(x, y)=$ $x\left(-a x^{k+1}+i(k+1) y\right)^{\beta} \in L_{l o c}^{p}\left(\boldsymbol{R}^{2}\right)$ for every $1 \leq p<-(k+2) /(1+(k+1) \operatorname{Re} \beta)$ if $\operatorname{Re} \beta<-1 /(k+1)$ and $F_{k, a, b}^{0, \beta, 1}(x, y) \in L_{l o c}^{\infty}\left(\boldsymbol{R}^{2}\right)$ if $\operatorname{Re} \beta \geq-1 /(k+1)$. It is easy to compute that 


$$
\begin{aligned}
&\left.B_{1}\left(F_{k, a, b}^{0, \beta, 1}, k, a, b,(k+1)(b-a)\right)\right|_{\rho=\varepsilon} \\
&= x^{k}\left(-a x^{k+1}+i(k+1) y\right)^{\beta-1} \times\left\{-((k+1)(b-a) \beta-a) x^{2 k+2}\right. \\
&+i(k+1)\left((k+1) a(b-a)(\beta+1)-a^{2}-1\right) x^{k+1} y+(k+1)^{2} \\
&\left.\times((k+1)(b-a)-a) y^{2}\right\}\left.\left(x^{4 k+2}+(k+1)^{2} y^{2}\right)^{-1 / 2}\right|_{\rho=\varepsilon}=\varepsilon^{2 k+1+(k+1) \beta} \\
& \times\left(-((k+1)(b-a) \beta-a) \sin ^{2} \theta+i\left((k+1) a(b-a)(\beta+1)-a^{2}-1\right)\right. \\
&\left.\times \cos \theta|\sin \theta|+((k+1)(b-a)-a) \cos ^{2} \theta\right)(\sin \theta)_{ \pm}^{k /(k+1)} \\
& \times(-a|\sin \theta|+i \cos \theta)^{\beta-1}\left(\varepsilon^{4 k+2}|\sin \theta|^{(4 k+2) /(k+1)}+\varepsilon^{2 k+2} \cos ^{2} \theta\right)^{-1 / 2}
\end{aligned}
$$

and

$$
\begin{aligned}
F_{k, a, b}^{0, \beta, 1} & \left.B_{2}(v, k, a, b) d s\right|_{\rho=\varepsilon} \\
& =-\frac{\varepsilon^{k+2+(k+1) \beta}}{k+1}|\sin \theta|^{1 /(k+1)}(-a|\sin \theta|+i \cos \theta)^{\beta}(|\sin \theta|-i b \cos \theta) X_{2} v(x, y) d \theta .
\end{aligned}
$$

Therefore we deduce that

$$
\begin{aligned}
-\int_{\rho=\varepsilon} & v(x, y) B_{1}\left(F_{k, a, b}^{0, \beta, 1}, k, a, b,(k+1)(b-a)\right) d s \\
& +\int_{\rho=\varepsilon} F_{k, a, b}^{0, \beta, 1}(x, y) B_{2}(v, k, a, b) d s \\
= & \frac{\varepsilon^{(k+1)(\beta+1)} v(0,0)}{k+1} \int_{-\pi}^{\pi}\left(-((k+1)(b-a) \beta-a) \sin ^{2} \theta\right. \\
& +i\left((k+1) a(b-a)(\beta+1)-a^{2}-1\right) \\
& \left.\times \cos \theta|\sin \theta|+((k+1)(b-a)-a) \cos ^{2} \theta\right) \operatorname{sign}(\sin \theta) \\
& \times(-a|\sin \theta|+i \cos \theta)^{\beta-1} d \theta+O\left(\varepsilon^{k+2+(k+1) \operatorname{Re} \beta}\right) .
\end{aligned}
$$

The integral in the right side of (12) vanishes for every $\varepsilon$ since its integrand is an odd function of $\theta$. Therefore using the assumption that $\operatorname{Re} \beta>-(k+2) /(k+1)$ we see that the expression in the left side of (12) tends to 0 as $\varepsilon$ tends to 0 . Hence $G_{k,(k+1)(b-a)}^{a, b} F_{k, a, b}^{0, \beta, 1}(x, y)=0$.

III) The proof of this part is the same as the proof of part II) with $-a, \beta$ replaced by $b, \alpha$.

IV) We note that if $k$ is odd and $\operatorname{Re} a<0$, and $\operatorname{Re} b<0$ then $\left(-b x^{k+1}+i(k+1) y\right)^{\alpha}$ and $\left(-a x^{k+1}+i(k+1) y\right)^{\beta} \in C^{\infty}\left(\boldsymbol{R}^{2} \backslash(0,0)\right)$ for every $\alpha$ and $\beta$. Therefore we can repeat all the arguments in part I). The only difference is 


$$
\begin{aligned}
-\int_{\rho=\varepsilon} & v(x, y) B_{1}\left(E_{k, a, b}^{\alpha_{2}, \beta_{2}, 1}, k, a, b, c\right) d s+\int_{\rho=\varepsilon} E_{k, a, b}^{\alpha_{2}, \beta_{2}, 1}(x, y) B_{2}(v, k, a, b) d s \\
\rightarrow & -\frac{2(c+b-a)(\partial v(0,0) / \partial x)}{k+1} \\
& \times \int_{0}^{\pi} \sin ^{1 /(k+1)} \theta(-a|\sin \theta|+i \cos \theta)^{\beta_{2}-1}(-b|\sin \theta|+i \cos \theta)^{\alpha_{2}} d \theta .
\end{aligned}
$$

Applying Corollary 2 with $\omega_{1}=\beta_{2}-1=-(c+(k+2)(b-a)) /((k+1)(b-a))$, $\omega_{2}=\alpha_{2}=(c-(k+1)(b-a)) /((k+1)(b-a)), \quad \omega_{3}=-1 /(k+1), \quad w_{1}=-a$, $w_{2}=-b$ yields

$$
\begin{aligned}
& -\frac{2(c+b-a)(\partial v(0,0) / \partial x)}{k+1} \\
& \quad \times \int_{0}^{\pi} \sin ^{1 /(k+1)} \theta(-a|\sin \theta|+i \cos \theta)^{\beta_{2}-1}(-b|\sin \theta|+i \cos \theta)^{\alpha_{2}} d \theta=0 .
\end{aligned}
$$

Now clearly we have $G_{k, c}^{a, b} E_{k, a, b}^{\alpha_{1}, \beta_{1}, 0}(x, y)=0$.

V) Argue as in part I) we arrive at

$$
\begin{gathered}
-\int_{\rho=\varepsilon} v(x, y) B_{1}\left(R_{k, a}^{\kappa_{2}, \eta_{2}, 1}, k, a, a, c\right) d s+\int_{\rho=\varepsilon} R_{k, a}^{\kappa_{2}, \eta_{2}, 1}(x, y) B_{2}(v, k, a, a) d s \\
\rightarrow-\frac{2 c(\partial v(0,0) / \partial x)}{k+1} \int_{0}^{\pi} \sin ^{1 /(k+1)} \theta \\
\quad \times(-a \sin \theta+i \cos \theta)^{\eta_{2}-1} e^{\kappa_{2} \sin \theta /(-a \sin \theta+\imath \cos \theta)} d \theta=0 .
\end{gathered}
$$

The last equality is a consequence of (13) when we let $b \rightarrow a$. Therefore we conclude that $G_{k, c}^{a, a} R_{k, a}^{\kappa_{2}, \eta_{2}, 1}(x, y)=0$. This concludes the proof of Theorem 2 .

COROLlaRY 4. If either $\operatorname{Re} a<0, \operatorname{Re} b>0, c=-((k+1) N+1)(b-a)$ or $\operatorname{Re} a<0, \operatorname{Re} b>0, c=(k+1)(b-a)(N+1)$ where $N$ is a non-negative integer, or $\operatorname{Re} a<0, \operatorname{Re} b<0$, then $G_{k, c}^{a, b}$ is not hypoelliptic (nor analytic hypoelliptic).

Proof. Indeed, if either $\operatorname{Re} a<0, \operatorname{Re} b>0, c=-((k+1) N+1)(b-a)$ or $\operatorname{Re} a<0, \quad \operatorname{Re} b>0, \quad c=(k+1)(b-a)(N+1) \quad$ then $\Gamma(((k+1)(b-a)-c) /$ $((k+1)(b-a)))=\infty \quad$ or $\quad \Gamma((c+b-a) /((k+1)(b-a)))=\infty \Rightarrow B_{k, c}^{a, b}=0 \Rightarrow$ $G_{k, c}^{a, b} F_{k, a, b}^{\alpha_{2}, \beta_{2}, 1}(x, y)=0$. If $\operatorname{Re} a<0, \operatorname{Re} b<0$, then $G_{k, c}^{a, b} E_{k, a, b}^{\alpha_{2}, \beta_{2}, 1}(x, y)=0$ in the non-resonance case or $G_{k, c}^{a, a} R_{k, a}^{\kappa_{2}, \eta_{2}, 1}(x, y)=0$ in the resonance case.

THEOREM 3. Assume that $k$ is even and $\operatorname{Re} a<0, \operatorname{Re} b>0$. If $c=(k+1)(b-a) N+((2 k+1)(b-a) / 2)$, where $N$ is an integer, then

$$
G_{k, c}^{a, b} F_{k, a, b}^{\alpha_{1}, \beta_{1}, 0}(x, y)=0, \quad G_{k, c}^{a, b} F_{k, a, b}^{\alpha_{2}, \beta_{2}, 1}(x, y)=0 .
$$


If $c=(k+1)(b-a) N+(k(b-a) / 2)$, where $N$ is an integer, then

$$
G_{k, c}^{a, b} F_{k, a, b}^{\alpha_{1}, \beta_{1}, 0}(x, y)=A_{k, c}^{a, b} \delta(x, y), \quad G_{k, c}^{a, b} F_{k, a, b}^{\alpha_{2}, \beta_{2}, 1}(x, y)=B_{k, c}^{a, b}(\partial \delta(x, y) / \partial x) .
$$

Proof. If $c=(k+1)(b-a) N+((2 k+1)(b-a) / 2)$, or $c=(k+1)(b-a) N$ $+(k(b-a) / 2)$, then $(k /(k+1))+2 \beta_{1}$ and $((k+2) /(k+1))+2 \beta_{2}$ are integers. Therefore $F_{k, a, b}^{\alpha_{1}, \beta_{1}, 0}(x, y), \quad F_{k, a, b}^{\alpha_{2}, \beta_{2}, 1}(x, y) \in C^{\infty}\left(\boldsymbol{R}^{2} \backslash(0,0)\right)$. Again we have $F_{k, a, b}^{\alpha_{1}, \beta_{1}, 0}(x, y) \in L_{l o c}^{((k+2) / k)-\tau}\left(\boldsymbol{R}^{2}\right)$ and $F_{k, a, b}^{\alpha_{2}, \beta_{2}, 1}(x, y) \in L_{l o c}^{((k+2) /(k+1))-\tau}\left(\boldsymbol{R}^{2}\right)$ for any small positive $\tau$. First we prove the theorem for $F_{k, a, b}^{\alpha_{1}, \beta_{1}, 0}$. As in the proof of Theorem 1 we can show that

$$
\int_{\rho=\varepsilon} F_{k, a, b}^{\alpha_{1}, \beta_{1}, 0}(x, y) B_{2}(v, k, a, b) d s \rightarrow 0 \quad \text { as } \varepsilon \rightarrow 0 .
$$

Next we have

$$
\begin{aligned}
& -\int_{\rho=\varepsilon} v(x, y) B_{1}\left(F_{k, a, b}^{\alpha_{1}, \beta_{1}, 0}, k, a, b, c\right) d s \\
& =-\frac{c}{k+1} \int_{-\pi}^{\pi}(\sin \theta)_{ \pm}^{-1 /(k+1)}(-a \sin \theta+i \cos \theta)^{\beta_{1}-1} \\
& \quad \times(b \sin \theta-i \cos \theta)^{\alpha_{1}} v(\varepsilon, \theta) d \theta \\
& =-\frac{c}{k+1} \int_{-\pi}^{\pi}(v(0,0)+\bar{o}(1))(\sin \theta)_{ \pm}^{-1 /(k+1)} \\
& \quad \times(-a \sin \theta+i \cos \theta)^{\beta_{1}-1}(b \sin \theta-i \cos \theta)^{\alpha_{1}} d \theta \\
& =-\frac{c v(0,0)}{k+1} \int_{-\pi}^{\pi}(\sin \theta)_{ \pm}^{-1 /(k+1)}(-a \sin \theta+i \cos \theta)^{\beta_{1}-1} \\
& \quad \times(b \sin \theta-i \cos \theta)^{\alpha_{1}} d \theta+\bar{o}(1) .
\end{aligned}
$$

If $c=(k+1)(b-a) N+((2 k+1)(b-a) / 2)$ then the integrand in the right side of (14) changes sign when we replace $\theta$ by $\theta-\pi$. Therefore the integral vanishes. Hence $G_{k, c}^{a, b} F_{k, a, b}^{\alpha_{1}, \beta_{1}, 0}(x, y)=0$. If $c=(k+1)(b-a) N+(k(b-a) / 2)$ then it follows that

$$
\begin{aligned}
& -\frac{c v(0,0)}{k+1} \int_{-\pi}^{\pi}(\sin \theta)_{ \pm}^{-1 /(k+1)}(-a \sin \theta+i \cos \theta)^{\beta_{1}-1}(b \sin \theta-i \cos \theta)^{\alpha_{1}} d \theta \\
& \quad=-\frac{2 c v(0,0)}{k+1} \int_{0}^{\pi}(\sin \theta)^{-1 /(k+1)}(-a \sin \theta+i \cos \theta)^{\beta_{1}-1}(b \sin \theta-i \cos \theta)^{\alpha_{1}} d \theta \\
& \quad=A_{k, c}^{a, b} v(0,0) .
\end{aligned}
$$

Therefore $G_{k, c}^{a, b} F_{k, a, b}^{\alpha_{1}, \beta_{1}, 0}(x, y)=A_{k, c}^{a, b} \delta(x, y)$. 
Next we prove the theorem for $F_{k, a, b}^{\alpha_{2}, \beta_{2}, 1}(x, y)$. As in Theorem 2 we have

$$
\begin{aligned}
-\int_{\rho=\varepsilon} v(x, y) B_{1}\left(F_{k, a, b}^{\alpha_{2}, \beta_{2}, 1}, k, a, b, c\right) d s+\int_{\rho=\varepsilon} F_{k, a, b}^{\alpha_{2}, \beta_{2}, 1}(x, y) B_{2}(v, k, a, b) d s \rightarrow \\
=-\frac{(c+b-a)(\partial v(0,0) / \partial x)}{k+1} \\
\quad \times \int_{-\pi}^{\pi}(\sin \theta)_{ \pm}^{1 /(k+1)}(-a \sin \theta+i \cos \theta)^{\beta_{2}-1}(b \sin \theta-i \cos \theta)^{\alpha_{2}} d \theta .
\end{aligned}
$$

If $c=(k+1)(b-a) N+((2 k+1)(b-a) / 2)$ then the integrand in the right side of (15) changes sign when we replace $\theta$ by $\theta-\pi$, therefore the integral vanishes. Hence $G_{k, c}^{a, b} F_{k, a, b}^{\alpha_{2}, \beta_{2}, 1}(x, y)=0$. If $c=(k+1)(b-a) N+(k(b-a) / 2)$ then we deduce that

$$
\begin{aligned}
& -\frac{(c+b-a)(\partial v(0,0) / \partial x)}{k+1} \\
& \times \int_{-\pi}^{\pi}(\sin \theta)_{ \pm}^{1 /(k+1)}(-a \sin \theta+i \cos \theta)^{\beta_{2}-1}(b \sin \theta-i \cos \theta)^{\alpha_{2}} d \theta \\
& =-\frac{2(c+b-a)(\partial v(0,0) / \partial x)}{k+1} \\
& \times \int_{0}^{\pi}(\sin \theta)_{ \pm}^{1 /(k+1)}(-a \sin \theta+i \cos \theta)^{\beta_{2}-1}(b \sin \theta-i \cos \theta)^{\alpha_{2}} d \theta \\
& =-B_{k, c}^{a, b} \frac{\partial v(0,0)}{\partial x} \text {. }
\end{aligned}
$$

If follows that $G_{k, c}^{a, b} F_{k, a, b}^{\alpha_{2}, \beta_{2}, 1}(x, y)=B_{k, c}^{a, b}(\partial \delta(x, y) / \partial x)$.

Corollary 5. Assume that $k$ is even and $\operatorname{Re} a<0, \quad \operatorname{Re} b>0$. If $c=(k+1)(b-a) N+(((2 k+1)(b-a)) / 2)$, where $N$ is an integer, then $G_{k, c}^{a, b}$ is not hypoelliptic (nor analytic hypoelliptic).

Remark 1. Altogether Corollary 3, Corollary 4 and Corollary 5 give all the values $k, a, b, c$, where $G_{k, c}^{a, b}$ is not hypoelliptic, as stated in [1], [2].

Remark 2. Since $G_{k, c}^{a, b}$ is invariant under the translation $(x, y) \rightarrow\left(x, y+y_{0}\right)$ it is easy to have the fundamental solutions or singular solutions at points $\left(0, y_{0}\right)$ in all cases considered above.

\section{REFERENCES}

[1] A. Gilioli AND F Treves, An example in the solvability theory of linear PDE's, Amer. J. Math., 96 (1974), 367-385.

[2] A. Menikoff, Some examples of hypoelliptıc partıal differential equations, Math. Ann., 221 (1976), 176-181. 
[3] M. Mascarello, Su alcune funzıon speciali che intervengono nello studio dell'ipoellitticita, Rend. Sem. Mat. Univ. Politec. Torino 36 (1977-1978), 343-349.

[4] P Bolley, J. Camus, B. HelfFer, Sur une classe d'opérateurs partiellement hypoelliptıques, J. Math. Pures Appl., 55 (1976), 131-171.

[5] B. HelfFer and L. Rodino, Opérateurs différentiels ordinaires intervenant dans l'étude de l'hypo-elliptıcté, Boll. Un. Mat. Ital. B., 14 (1977), 491-522.

[6] P R. Popivanov, Construction of solutions of pseudo-differential equations with given singularities, C. R. Acad. Bulgare Scl., 34 (1981), 165-167

[7] M. Mascarello and L. Rodino, Partial differential Equations with Multiple Characteristics, Academie-Verlag, 1997

[8] N. M. TRI, Remark on non-uniform fundamental solutions and non-smooth solutions of some classes of differential operators with double characteristics, J. Math. Scı. Unıv. Tokyo, 6 (1999), 437-452.

[9] N. M. TRI, On the Gevrey regularity of solutions of semilinear Kohn-Laplacian on the Heisenberg group, Math. Note Institute of Math., Univ. Tsukuba (1998).

[10] P APPEL AND J. KAMPÉ DE FÉRIET, Functions Hypergémétriques et Hypersphérıques. Polynomes d'Hermite, Gauthier-Villars, 1926.

InSTITUTE OF MATHEMATICS

P.O. Box 631, BOHO 10000

HaNOI, ViETNAM 